\title{
Rotating Ayón-Beato-García black hole as a particle accelerator
}

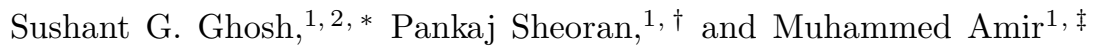 \\ ${ }^{1}$ Centre for Theoretical Physics, Jamia Millia Islamia, New Delhi 110025 India \\ ${ }^{2}$ Astrophysics and Cosmology Research Unit, School of Mathematics, \\ Statistics and Computer Science, University of KwaZulu-Natal, \\ Private Bag X54001, Durban 4000, South Africa
}

\begin{abstract}
We study the collision of two particles with equal masses moving in the equatorial plane near horizon of the rotating regular Ayón-Beato-García (ABG) black hole (BH) and calculate the centerof-mass (CM) energy for the colliding particles for both extremal and non-extremal cases. It turns out that CM energy depends not only on rotation parameter $a$ but also on charge $Q$. Particularly for the extremal rotating regular ABG BH, CM energy of two colliding particles could be arbitrarily high for critical angular momentum of particles. Furthermore, we also show that, for a non-extremal $\mathrm{BH}$, there exist a finite upper limit of $\mathrm{CM}$ energy, which changes with charge $Q$. A comparison, with Kerr and Kerr-Newman black holes, is included.

PACS numbers: 97.60.Lf, 04.70.-s, 04.70.Bw
\end{abstract}

\section{INTRODUCTION}

Recently, Banãdos-Silk-West (BSW) [1] studied collision of two particles (e.g. dark matter particles) in the vicinity of the horizon of the Kerr black hole $(\mathrm{BH})$ and found that center-of-mass (CM) energy of the colliding particles in the equatorial plane can be arbitrarily high in the limiting case of extremal $\mathrm{BH}$. This imply that the extremal rotating $\mathrm{BH}$ may be considered as a Planck energy scale particle accelerator, which might allow us to explore ultra high energy collisions and astrophysical phenomena, such as the gamma ray bursts and the active galactic nuclei. In the work of BSW [1], two particles of equal mass and equal energy falling freely from rest at infinity and approaching the extremal Kerr BH on the equatorial plane were considered. The energy in the $\mathrm{CM}$ frame was computed and the critical value of the particle angular momentum at which the energy blows up were determined. However, Jacobson and Sotiriou 2] elucidate the mechanism for this result, and point out its practical limitations given that extremal BHs do not exist in nature, e.g., the spin $a$ of astrophysical BHs should not exceed $a / M=0.998[3]$ ( $M$ is the mass of the $\mathrm{BH})$. In any case, the third law of $\mathrm{BH}$ thermodynamics (Bardeen et al. 1973) asserts that a $\mathrm{BH}$ cannot be spun-up in a finite time to the extreme spin value $a / M=1$. In particular, Jacobson and Sotiriou [2] demonstrated that infinite collision energy can only be attained at the horizon, and with a maximally spinning BH. Lake [4] also demonstrated that the CM energy of colliding particle near the inner horizon of a non-extremal Kerr BH is finite. It is known that the motion of a particle traveling in the background of a charged spinning $\mathrm{BH}$ depends not only on the spin but also on the charge of the BH. There-

\footnotetext{
*Electronic address: sghosh2@jmi.ac.in

${ }^{\dagger}$ Electronic address: hukmipankaj@gmail.com

${ }^{\ddagger}$ Electronic address: amirctp12@gmail.com
}

fore, the CM energy of collision expected to depend both on spin and charge. The BSW scenario is generalized to charged BHs [5], the Kerr-Newman family of BHs [6] and general rotating $\mathrm{BHs}$ [7]. It is shown that, for a nearextremal charged $\mathrm{BH}$, there always exists a finite upper bound for CM energy, which decreases with the increase of the charge $Q$.

The BSW effect near the event horizon of the KerrTaub-NUT BH was investigated by [8], around the Kaluza-Klein (extremal) $\mathrm{BH}$ in [9] and found the infinitely large CM energy near the horizon in both rotating and non-rotating cases. The BSW effect for the EinsteinMaxwell-dilaton-axion BH [10], and the BTZ BH was discussed in [11]. In [12], the CM energy was generalized for charged particles moving in an electromagnetic field and braneworld BHs were discussed. Even for nonmaximally rotating BHs, Grib and Pavlov [13 16] argued that the CM energy for two particles collision can be unlimited for the non-maximal rotation as well when one considers the multiple scattering. A general explanation for the arbitrarily high CM energy is presented in terms of the Killing vectors and the Killing horizon by Zaslavskii [17]. Further, the author [18 20] clarified that the universal property of acceleration of particles near rotating BHs and give a general argument of this BSW mechanism for the general rotating BHs. Further, Joshi and Patil 21] found that the CM energy to be high for the naked singularity of the Kerr BH and other BHs [22].

More recently, BSW mechanism is used to calculate the CM energy of collision for two particles freely falling, from rest at infinity, in the horizon of a Ayón-BeatoGarcía (ABG) BH [23]. It turns out that the CM energy for $\mathrm{ABG} B \mathrm{BH}$ can be infinitely high for the extremal case [23]. The rotating counterpart of ABG BH is another interesting and important spacetime [24], which is a solution of Einstein equations coupled to nonlinear electrodynamics. Besides the mass $\mathrm{M}$ and the rotation parameter $a$, the rotating $\mathrm{ABG}$ spacetime carries with the charge, $Q$. It is widely believed that spacetime singularities do not exist in Nature, but that they represent a limitation 
of the classical theory. While we do not yet have any solid theory of quantum gravity models of $\mathrm{BH}$ solutions without singularities have been proposed [25 27]. These spacetimes have an event horizon and no pathological features like singularities or regions with closed timelike curves. The rotating ABG metrics are more important as they can be tested by astrophysical observations, as the $\mathrm{BH}$ spin plays a fundamental role in any astrophysical process 28]. The rotating ABG black hole are axisymmetric, asymptotically flat and depend on the mass and spin of the black hole as well as on a parameter $Q$ that measure potential deviations from the Kerr metric. The rotating ABG metric includes the Kerr metric as the special case if this deviation parameter vanishes.

The main purpose of this paper is to study the collision of two particles in the background of the rotating $\mathrm{ABG}$ spacetime and to see what the effects of the charge $Q$ on the CM energy for the particles in the near-horizon collision. It turns out that our results can be reduced to the ones of BSW [1] as the charge parameter turns to zero and nonrotating $\mathrm{ABG} \mathrm{BH}$ when the rotation parameter $a=0$. Besides, we may be more interest to discuss CM energy of the rotating $\mathrm{ABG} \mathrm{BH}$ because it does not have a simple horizon structure as the previous studied BHs.

\section{ROTATING AYÓN-BEATO-GARCÍA BLACK HOLE}

In this section, we would like to study the rotating ABG BHs. The gravitational field of rotating ABG spacetime [24] is described by metric which in the BoyerLindquist coordinates with units $c=G=1$ is given by

$$
\begin{aligned}
d s^{2}= & -f(r, \theta) d t^{2}+\frac{\Sigma}{\Delta} d r^{2} \\
& -2 a \sin ^{2} \theta(1-f(r, \theta)) d \phi d t+\Sigma d \theta^{2} \\
& +\sin ^{2} \theta\left[\Sigma-a^{2}(f(r, \theta)-2) \sin ^{2} \theta\right] d \phi^{2}
\end{aligned}
$$

where the function $f(r, \theta)$ and $\Delta$ are given by

$$
\begin{aligned}
f(r, \theta) & =1-\frac{2 M r \sqrt{\Sigma}}{\left(\Sigma+Q^{2}\right)^{3 / 2}}+\frac{Q^{2} \Sigma}{\left(\Sigma+Q^{2}\right)^{2}}, \\
\Delta & =\Sigma f(r, \theta)+a^{2} \sin ^{2} \theta
\end{aligned}
$$

and $\Sigma=r^{2}+a^{2} \cos ^{2} \theta$. The parameters $a, M$ and $Q$ are respectively correspond to rotation, mass and the electric charge. We shall demonstrate that, for certain range of values of $M$ and $Q$, the metric (1) is a BH. The curvature invariant $R, R_{a b} R^{a b}$ and $R_{a b c d} R^{a b c d}$ for the metric (1) reveals that the rotating $\mathrm{ABG} \mathrm{BH}$ is regular everywhere for $a, M, Q \neq 0$. The metric (1) is a non-singular rotating charged $\mathrm{BH}$ which generalizes the standard Kerr BH. In addition, if $Q=0$ the metric (1) reduce to Kerr $\mathrm{BH}$ [29]. Further when both $a, Q=0$ the metric (11) is Schwarzchild BH [30]. If $a \rightarrow 0$ we have an exact ABG $\mathrm{BH}$ [31] which is a solution of Einstein field equations coupled to the nonlinear electrodynamics. We know that the Kerr BH has two horizons like surfaces: static limit surface and event horizon. We are interested in these two surfaces for the rotating ABG spacetime metric (1). We start with calculating location and structure of static limit surface which requires the prefactor of $d t^{2}$ to vanish. Then it follows from (11) that the static limit surface will satisfy

$$
f(r, \theta)=\left(\Sigma+Q^{2}\right)^{2}-2 M r \sqrt{\Sigma\left(\Sigma+Q^{2}\right)}+Q^{2} \Sigma=0 .
$$

The Eq. (3) for $Q=0$ becomes exactly same as Kerr [29]. The surface of no return is known as the event horizon. Beyond event horizon the gravitational force becomes so high that it is impossible for any object, even light to escape from the gravity of the $\mathrm{BH}$. The spacetime (1) generically must have two horizons, viz., the inner or Cauchy horizon and the outer or event horizon. The horizons of the BH (11) are calculated by equating the $g^{r r}$ component of the metric (11) to zero, i.e.,

$$
\Delta=\Sigma f(r, \theta)+a^{2} \sin ^{2} \theta=0,
$$

which leads to

$$
\begin{array}{r}
\Sigma\left(\Sigma+Q^{2}\right)^{2}-2 M r \Sigma^{3 / 2}\left(\Sigma+Q^{2}\right)^{1 / 2}+Q^{2} \Sigma^{2} \\
+a^{2}\left(\Sigma+Q^{2}\right)^{2} \sin ^{2} \theta=0 .
\end{array}
$$

Clearly, the radii of the horizons depends on $\theta$, which are different from that in the usual Kerr case. The behavior of the static limit surface is shown in Fig. 1 and that of event horizon in Fig. 24 for different values of mass $M$, charge $Q$ and spinning parameter $a$. The two figures reveals that there exists set of values of parameters for which we have two horizons, i.e., a regular $\mathrm{BH}$ with both inner and outer horizons. One can also find values of parameters for which one get extreme BHs where the two horizons are coincides. The region between the static limit surface and the event horizon is termed as quantum ergosphere. The ergosphere is the region which lies outside the $\mathrm{BH}$. In ergosphere it is possible to enter and leave again, and the object moves in the direction of the spin of $\mathrm{BH}$.

\section{PARTICLE ORBITS IN THE ROTATING AYÓN-BEATO-GARCÍA BLACK HOLE}

Astrophysical $\mathrm{BH}$ candidates are supposed to be the Kerr BHs as predicted in general relativity, but the actual nature of these objects has still to be substantiated 32 34]. If one wish to test the nature of an astrophysical $\mathrm{BH}$ candidate, it is better to consider a more general spacetime, like rotating $\mathrm{ABG} \mathrm{BH}$, than a Kerr, in which the central object is described by a mass $M$, spin parameter $a$, and an additional deviation parameter $Q$. The rotating ABG metric can be seen as the prototype of a large class of non-Kerr BH metrics and the Kerr solution must be recovered when this deformation parameter $Q$ vanishes (cf. Fig. 5). If the observations require vanishing deformation parameter, the compact object is a 

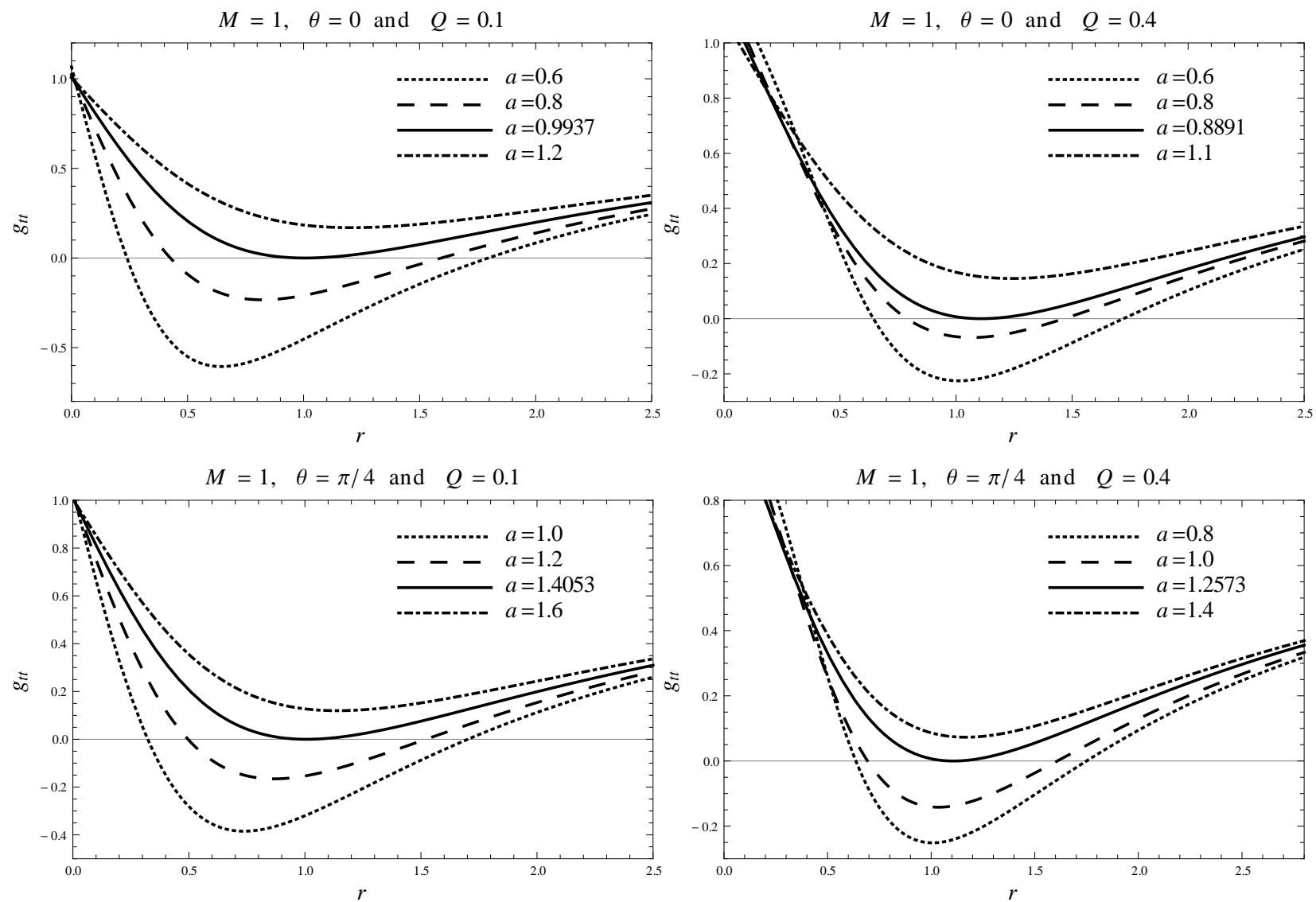

FIG. 1: The behavior of static limit surface $\left(g_{t t}\right)$ vs $r$ for different values of $a$. The possibility of existence of horizon is decreases with increases values of $Q$ and $a$.
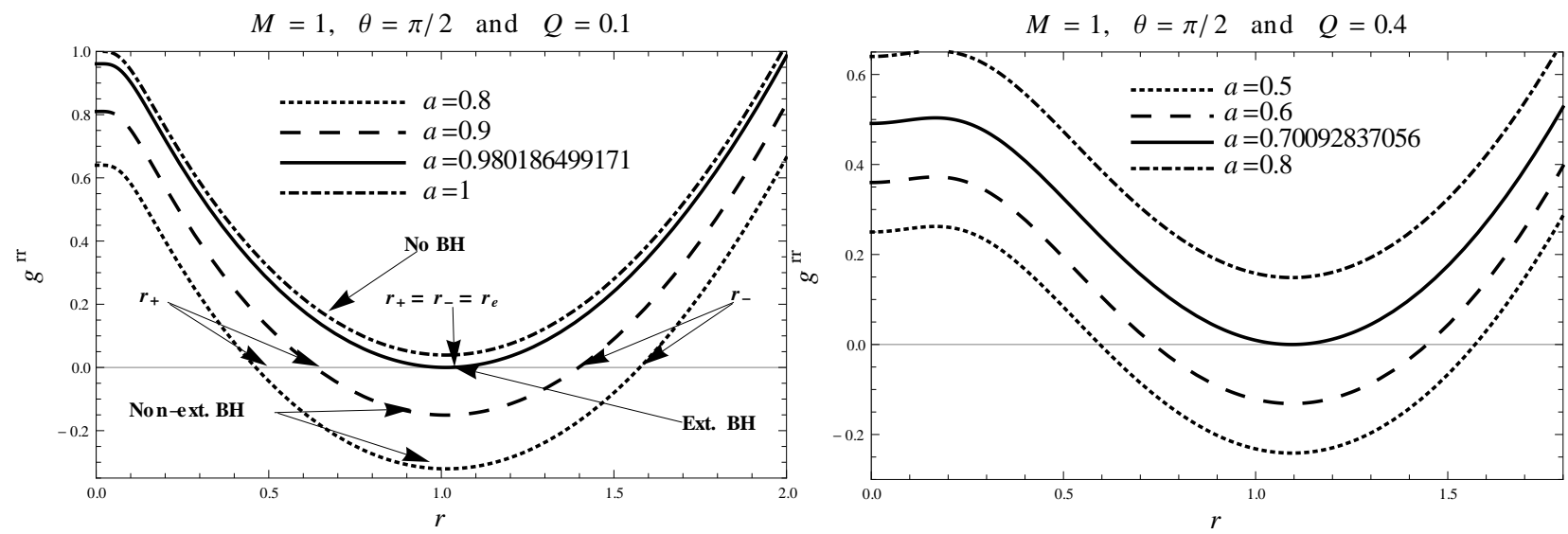

FIG. 2: The behavior of $g^{r r}$ vs $r$ for different values of $a$. Panel (a) for $Q=0.1$. Panel (b) for $Q=0.4$.

Kerr BH or non-Kerr BH otherwise. In general, observations allows both the possibility of a Kerr $\mathrm{BH}$ with a certain spin parameter and non-Kerr BH with different spin parameters. Further, we are far from a reliable candidate for a quantum theory of gravity, hence recently more attention is given for phenomenological approaches to somehow solve these singularity problem in classical general relativity and study possible implications. In this context, an important line of research is represented by the work on the regular BH solutions Hence, it pertinent to consider BSW mechanism for the rotating ABG black holes.

Let us consider a motion for a particle with mass $m_{0}$ falling in the background of a rotating $\mathrm{ABG} \mathrm{BH}$. The 

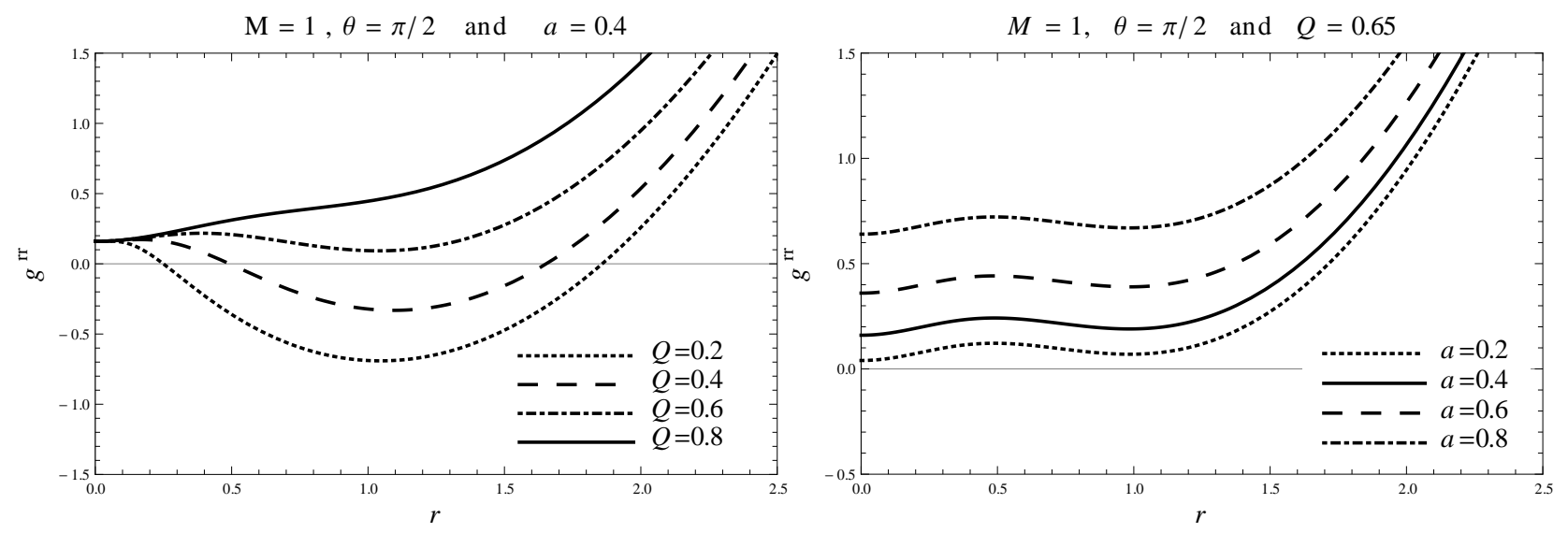

FIG. 3: The behavior of $g^{r r}$ vs $r$. Panel (a) for fixed $a=0.4$ and different values of $Q$. Panel (b) for fixed $Q=0.65$ and different values of $a$.
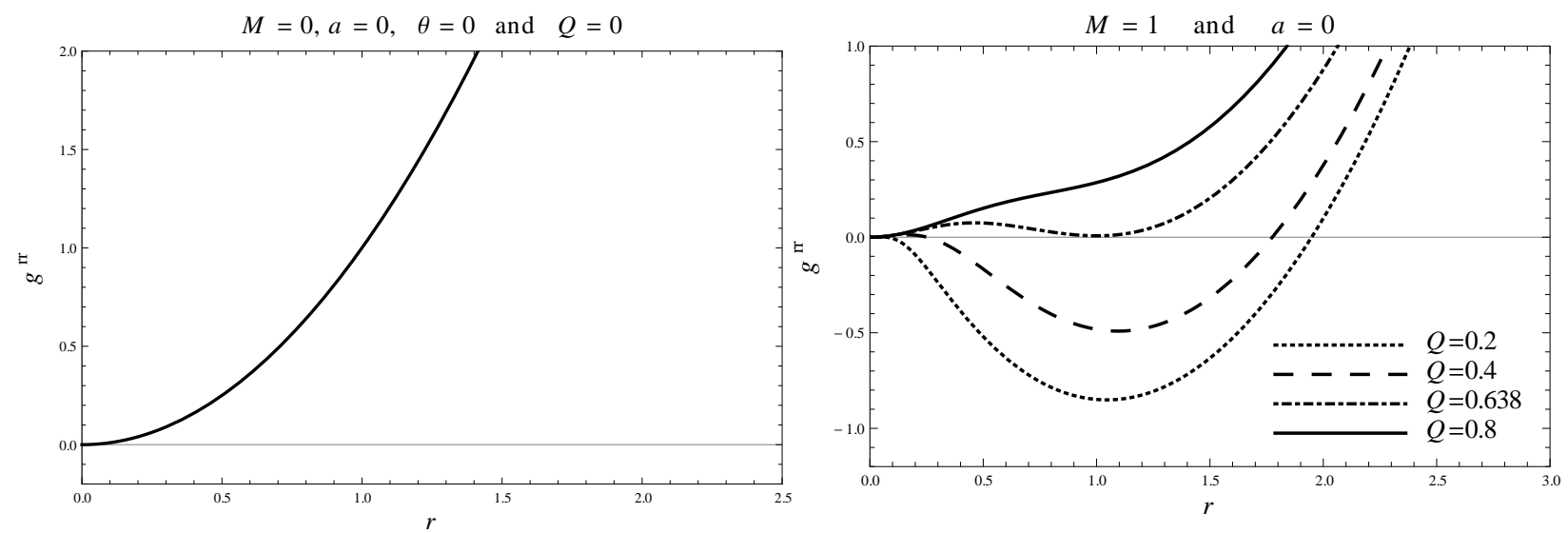

FIG. 4: The behavior of $g^{r r}$ vs $r$. Panel (a) for $a=M=Q=0$. Panel (b) for $M=1, a=0$ and different values of $Q$.

geodesic motion for this $\mathrm{BH}$ is determined by the following Hamilton-Jacobi equation

$$
\frac{\partial S}{\partial \tau}=-\frac{1}{2} g^{\mu \nu} \frac{\partial S}{\partial x^{\mu}} \frac{\partial S}{\partial x^{\nu}},
$$

where $\tau$ is an affine parameter along the geodesics, and $S$ is the Jacobi action. For this BH background the Jacobi action $S$ can be separated as

$$
S=\frac{1}{2} m_{0}^{2} \tau-E t+L \phi+S_{r}(r)+S_{\theta}(\theta)
$$

where $S_{r}$ and $S_{\theta}$ are function of $r$ and $\theta$ respectively. The constants $m_{0}, E$, and $L$ correspond to rest mass, conserved energy and angular momentum through $m_{0}^{2}=$ $-p_{\mu} p^{\mu}, E=-p_{t}$, and $L=p_{\phi}$. For equatorial plane $(\theta=$ $\pi / 2)$ in the ABG metric (11), we obtain the null geodesics in the form of the first-order differential equations

$$
\begin{gathered}
\frac{d t}{d \tau}=\frac{1}{r^{2}}\left[-a(a E-L)+\left(r^{2}+a^{2}\right) \frac{T}{\Delta}\right], \\
\frac{d \phi}{d \tau}=\frac{1}{r^{2}}\left[-(a E-L)+\frac{a T}{\Delta}\right],
\end{gathered}
$$

$$
\frac{d r}{d \tau}= \pm \frac{1}{r^{2}} \sqrt{T^{2}-\Delta\left[m_{0}^{2} r^{2}+(L-a E)^{2}+K\right]},
$$

where $T=E\left(r^{2}+a^{2}\right)-L a$ and $K$ is a constant. These equations determine the propagation of light in the spacetime of the rotating $\mathrm{ABG} \mathrm{BH}$. Obviously, for $Q=0$, it is just the null geodesic for the Kerr BH. The constant $K=0$ is the necessary and sufficient condition for particles motion initially in the equatorial plane to remain in the equatorial plane. Any particle which crosses the equatorial plane has $K>0$ [35].

The study of effective potential is a very useful tool for describing the motion of test particles. The radial equation for timelike particles moving along geodesic in the equatorial plane $(\theta=\pi / 2)$ is described by

$$
\frac{1}{2} \dot{r}^{2}+V_{e f f}=0,
$$

with the effective potential

$$
V_{\text {eff }}=-\frac{\left[E\left(r^{2}+a^{2}\right)-L a\right]^{2}-\Delta\left[m_{0}^{2} r^{2}+(L-a E)^{2}\right]}{2 r^{4}},
$$




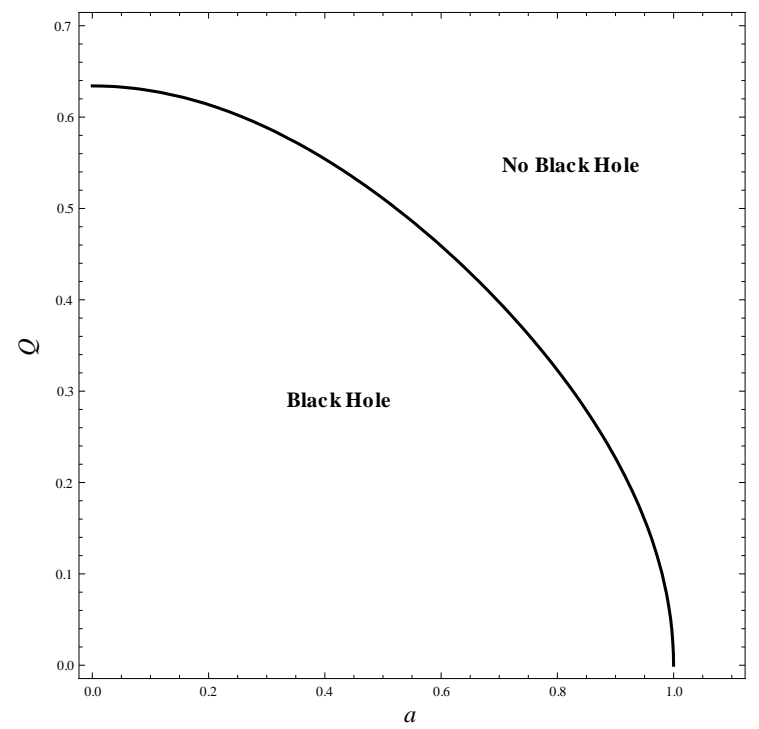

FIG. 5: Plot showing spin deformation parameter plane of rotating ABG metric. The curve separates $\mathrm{BH}$ from no $\mathrm{BH}$ or configuration without an event horizon (i.e., no real root of $\Delta=0)$.

The circular orbit for the particle is defined as

$$
V_{e f f}=0 \quad \text { and } \quad \frac{d V_{e f f}}{d r}=0,
$$

Eq. (13) leads to the limitation on the possible values of angular momentum of free falling particle and to achieve the horizon of the $\mathrm{BH}$ the angular momentum $L$ must be lying within the range $-4.80898 \lesssim L \lesssim 2.02893$ for extremal case. For extremal case $a \approx 0.980186499171$ and $r_{e} \approx 1.01388$ is event horizon. Whereas for the case of Kerr-Newman BH the extremal value of spin parameters is $a \approx 0.9$, range of angular momentum is $-4.6864 \lesssim L \lesssim 2.0111$ and the $r_{e} \approx 1$.

Since, $u^{t}=d t / d \tau \geq 0$, then from Eq. (8) the condition

$$
\begin{gathered}
E\left[\left(r^{2}+a^{2}\right)\left(r^{2}+Q^{2}\right)^{2}+2 M r^{2} a^{2} \sqrt{r^{2}+Q^{2}}-Q^{2} r^{2} a^{2}\right] \\
\geq \operatorname{Lar}^{2}\left[2 M \sqrt{r^{2}+Q^{2}}-Q^{2}\right],
\end{gathered}
$$

must be satisfied, as $r \rightarrow r_{+}$, this condition reduces to

$$
E \geq \frac{a L}{r_{+}^{2}+a^{2}}=\Omega_{+} L .
$$

\section{CENTER OF MASS ENERGY OF TWO COLLIDING PARTICLES}

We have dealt with range of the angular momentum, for which the particle can reach the horizon, i.e., if the angular momentum of particles are in the desired range, the collision can take place near horizon of the $\mathrm{BH}$. We are now in the position to study the properties of the CM energy of two colliding particles with same rest mass $m_{0}$ near horizon of regular $\mathrm{ABG} \mathrm{BH}$.

Next, We study CM energy of the collision of two particles moving in equatorial plane of rotating $\mathrm{ABG} \mathrm{BH}$. Let us consider two colliding particles with the same rest mass $m_{1}=m_{2}=m_{0}\left(E=m_{0}\right)$ are at rest at infinity. They are coming from rest at infinity and approaching the $\mathrm{BH}$ with different angular momenta $L_{1}$ and $L_{2}$ and collide at some radius $r$. Our aim is to compute the energy in CM frame for this collision. We assume that two particles 1 and 2 are at same point with four momentum

$$
p_{i}^{\mu}=m_{0} u_{i}^{\mu},
$$

where $p_{i}^{\mu}$ and $u_{i}^{\mu}$ are the four momentum and four velocity of the $i-t h$ particle $(i=1,2)$. The sum of 2 -momenta is given as

$$
p_{\text {tot }}^{\mu}=p_{1}^{\mu}+p_{2}^{\mu},
$$

The CM energy of two-particles is given by

$$
E_{C M}^{2}=m_{1}^{2}+m_{2}^{2}-2 g_{\mu \nu} p_{1}^{\mu} p_{2}^{\nu},
$$

Clearly, $E_{C M}$ is scalar. The formula (18) is valid for both massless and massive particles, and independent of coordinate choice. In the CM energy case of equal mass $m_{1}=m_{2}=m_{0}$ the formula (18) reduces to

$$
E_{C M}=m_{0} \sqrt{2} \sqrt{1-g_{\mu \nu} u_{(1)}^{\mu} u_{(2)}^{\nu}},
$$

Here we assume that the 2-particles coming from infinity with $E_{1} / m_{0}=E_{2} / m_{0}=1$, for simplicity. The CM energy of two equal mass particles in the rotating $\mathrm{ABG}$ spacetime is given by 

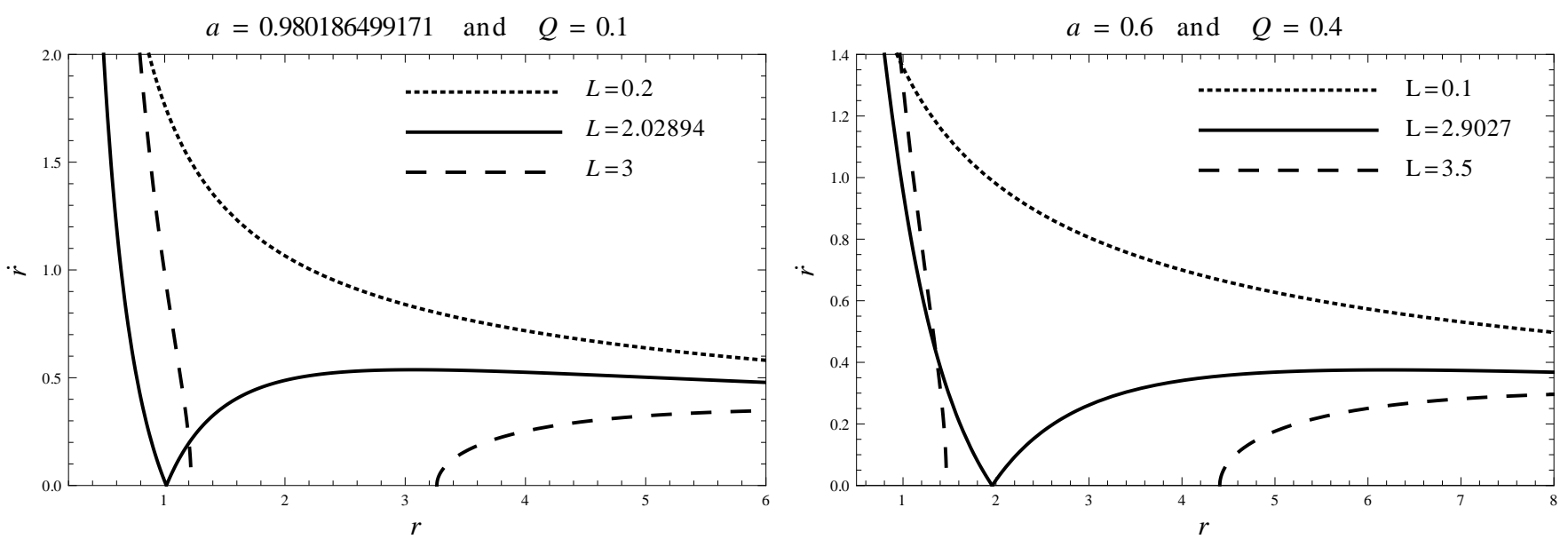

FIG. 6: The behavior of $\dot{r}$ vs $r$ for different values of $L$. Panel (a) for extremal BH. Panel (b) for non-extremal BH.
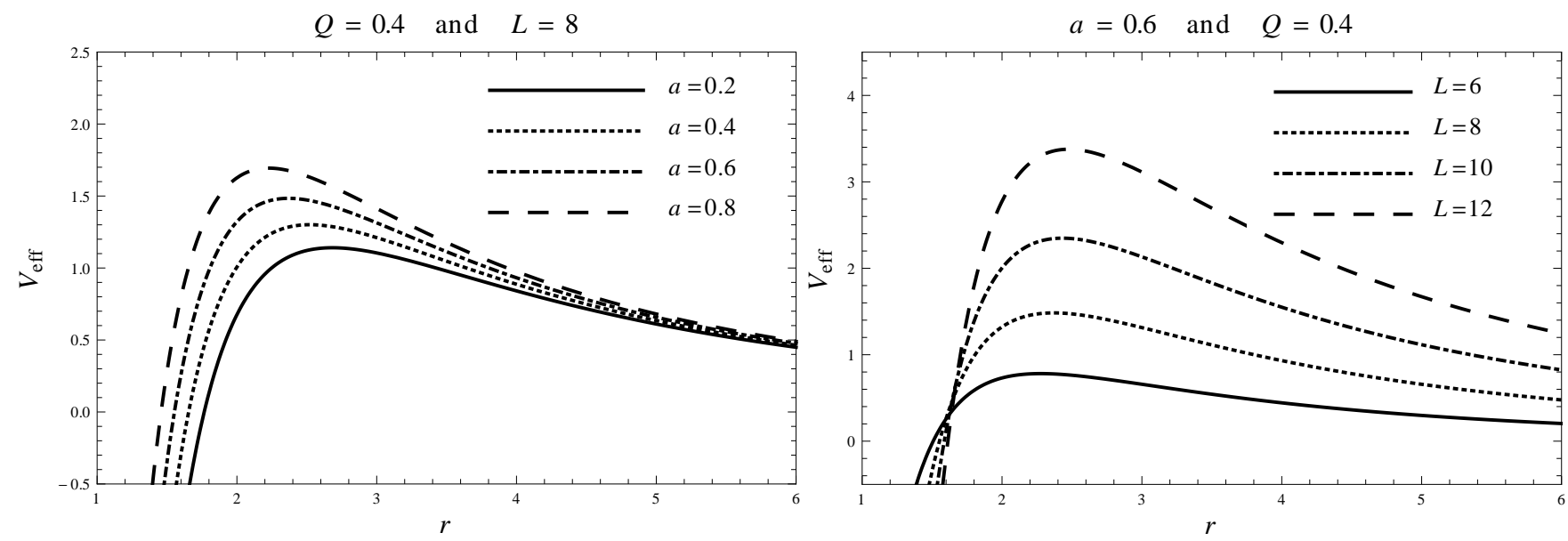

FIG. 7: The behavior of $V_{\text {eff }}$ vs $r$. Panel (a) for $Q=0.4, L=8$ and different values of $a$. Panel (b) for $Q=0.4, a=0.6$ and different values of $L$.
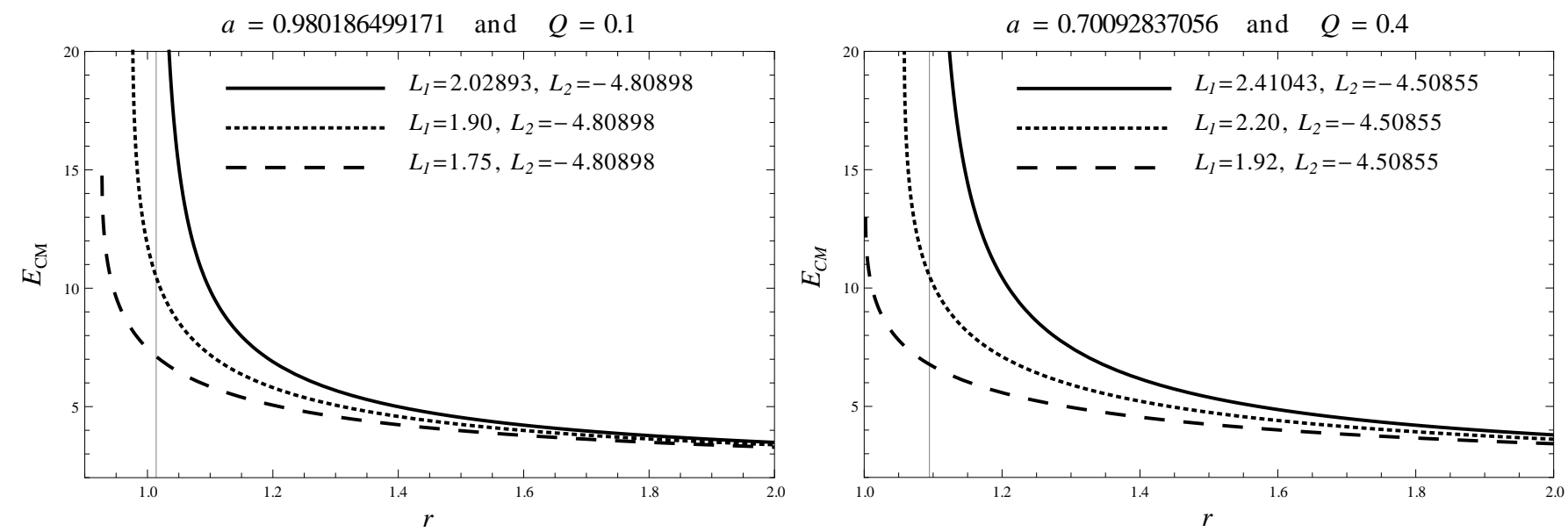

FIG. 8: The behavior of $E_{C M}$ vs $r$ for extremal BH. Panel (a) for $a=0.980186499171$ and $Q=0.1$. Panel (b) for extremal values of $a=0.70092837056$ and $Q=0.4$ where the vertical lines denote the location of event horizon. 

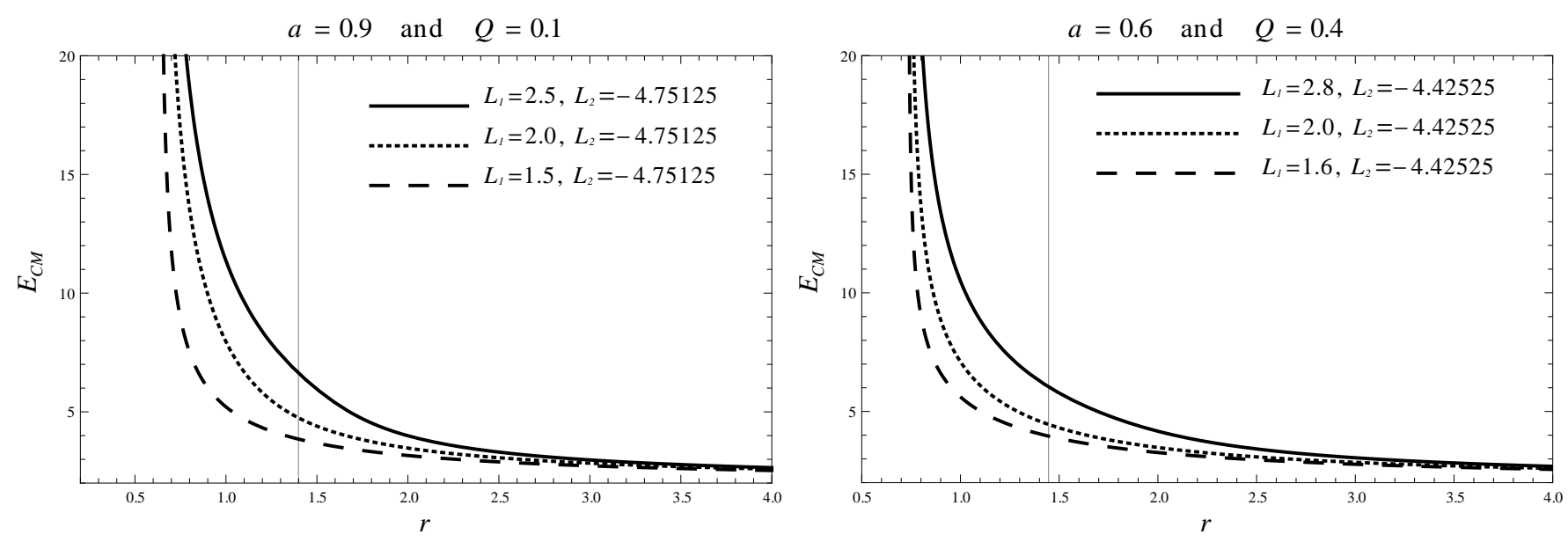

FIG. 9: The behavior of $E_{C M}$ vs $r$ for non-extremal BH. Panel (a) for $a=0.9$ and $Q=0.1$. Panel (b) for $a=0.6$ and $Q=0.4$ where the vertical lines denote the location of the event horizon.
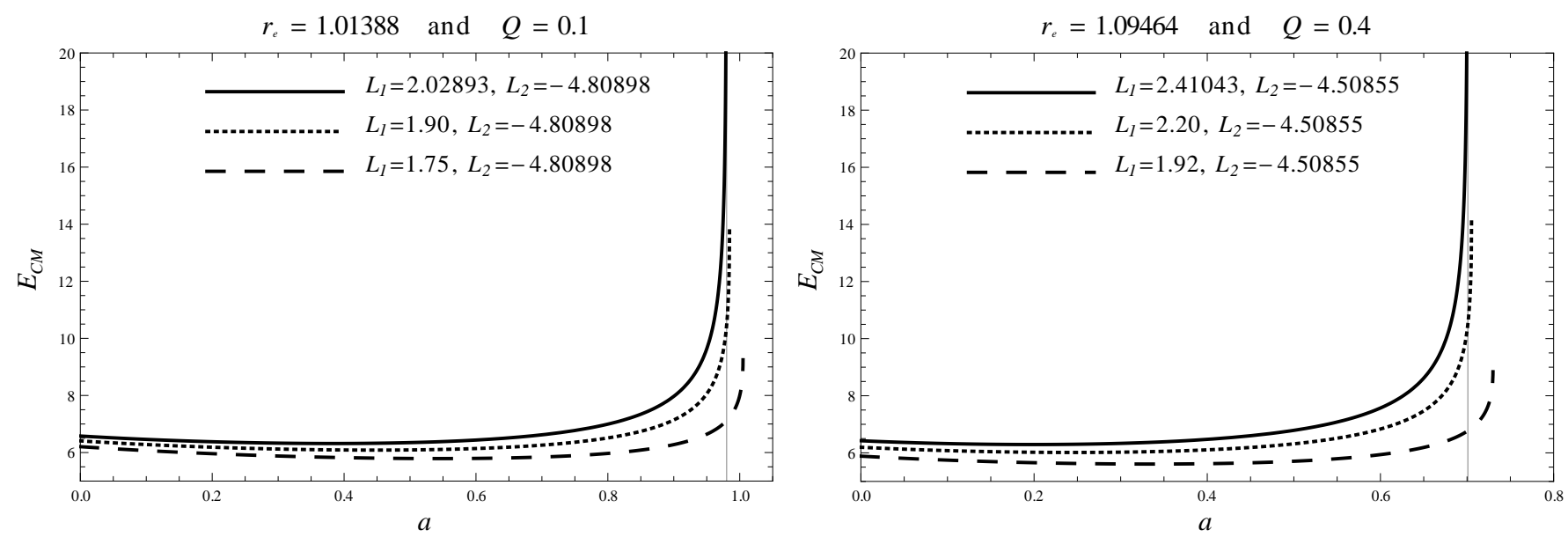

FIG. 10: The behavior of $E_{C M}$ vs a for extremal BH. Panel (a) for different values of $L_{1}$ where vertical line denotes the location of $a=0.980186499171$. Panel (b) for different values of $L_{1}$ where vertical line denotes the location of $a=0.70092837056$.
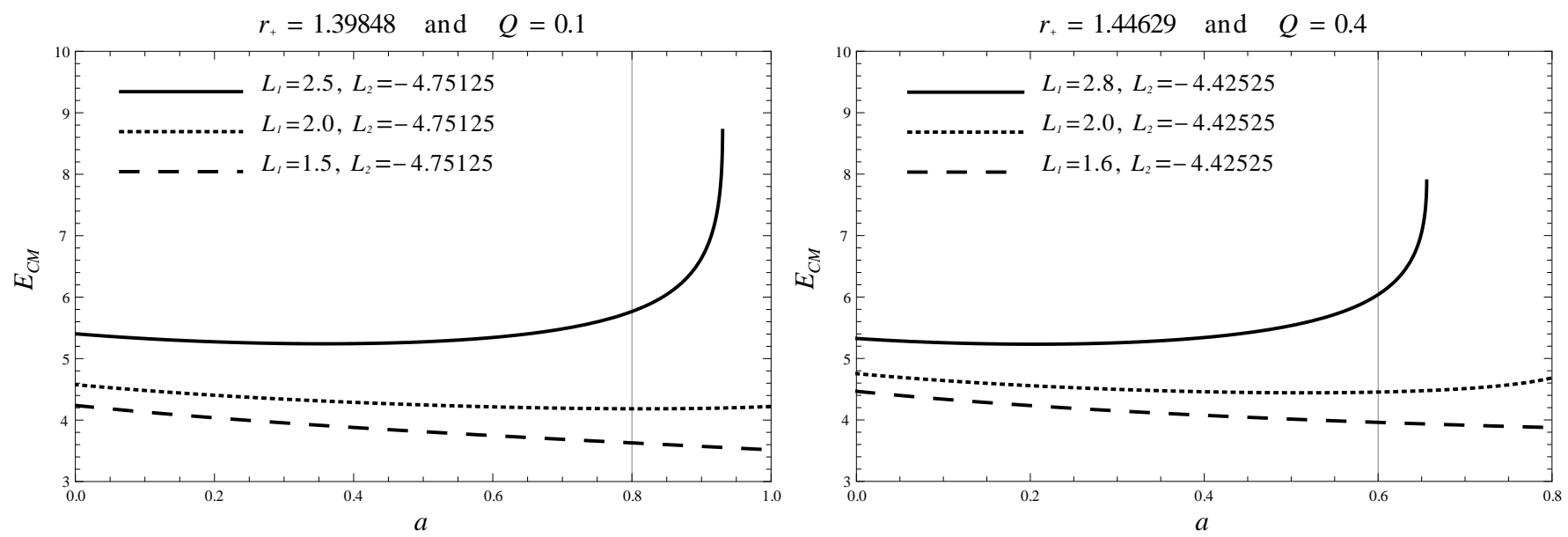

FIG. 11: The behavior of $E_{C M}$ vs $a$ for non-extremal BH. Panel (a) for different values of $L_{1}$ where vertical line denotes the location of $a=0.9$. Panel (b) for different values of $L_{1}$ where vertical line denotes the location of $a=0.6$. 

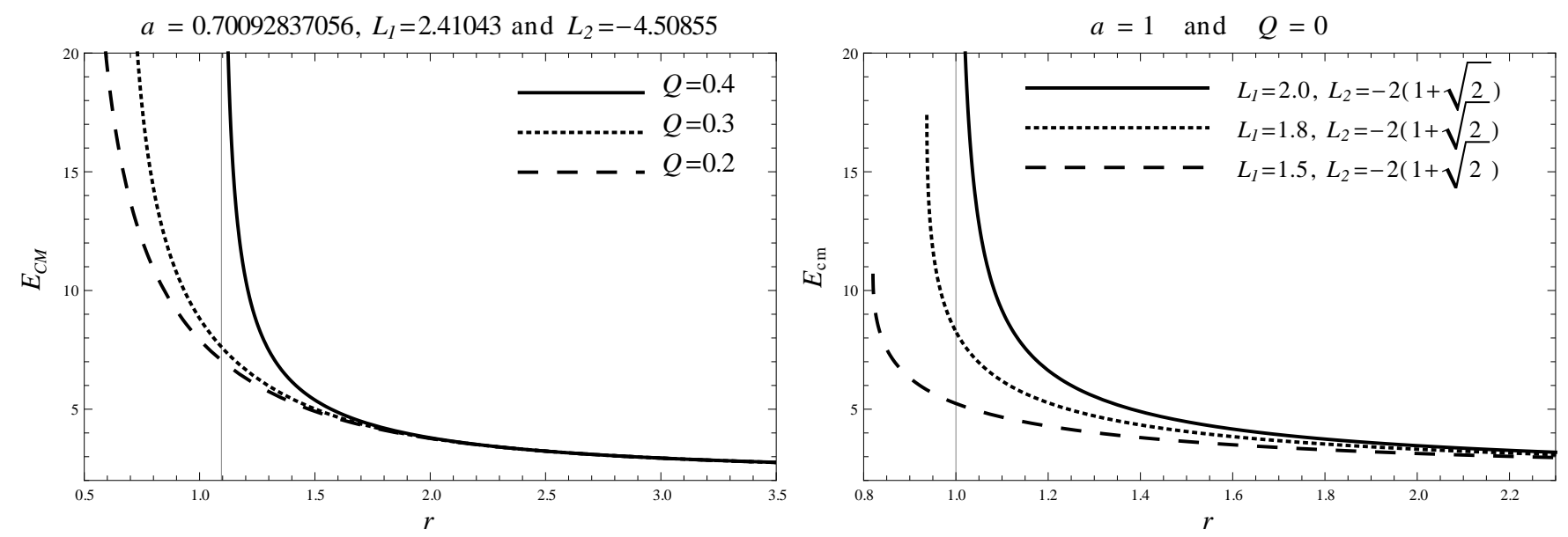

FIG. 12: The behavior of $E_{C M}$ vs $r$. Panel (a) for different values of $Q$ where vertical line denotes the location of $r=1.09464$. Panel (b) for extremal Kerr BH where vertical line denotes the location of event horizon.
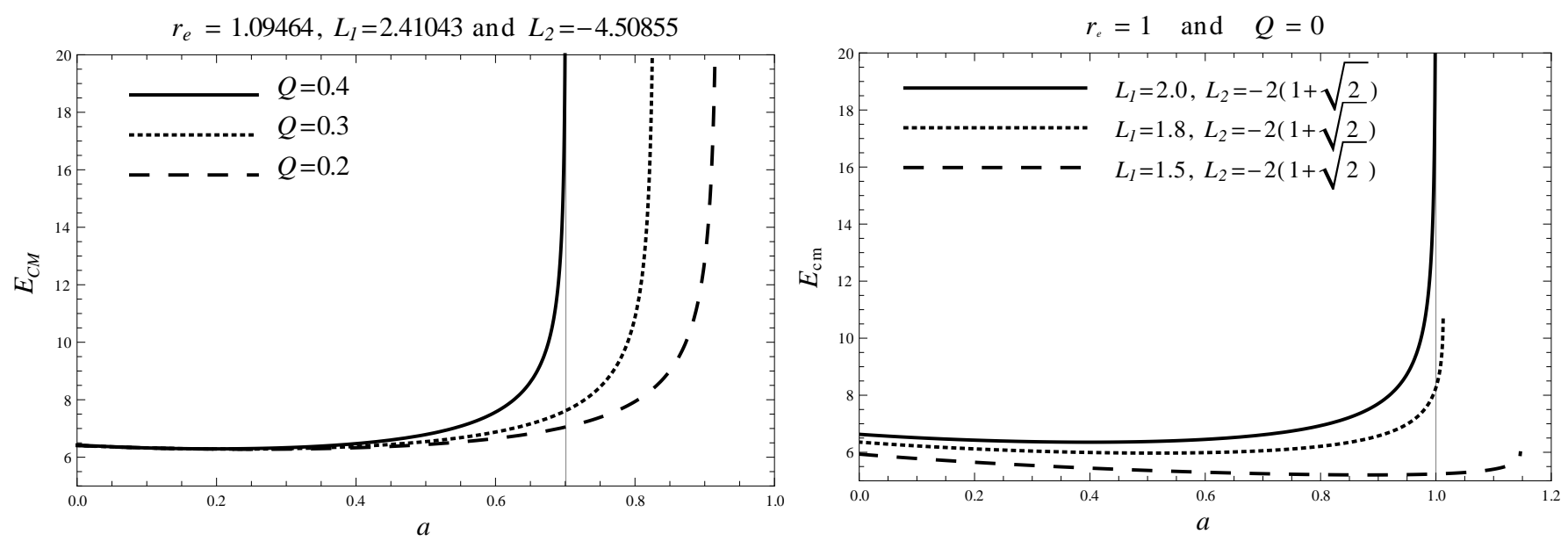

FIG. 13: The behavior of $E_{C M}$ vs $a$. Panel (a) for different values of $Q$ where vertical line denotes the location of $a=$ 0.70092837056. Panel (b) for extremal Kerr BH where vertical line denotes the location of $a=1$.

$$
\begin{aligned}
\frac{E_{C M}^{2}}{2 m_{0}^{2}} & =\frac{1}{r^{2}\left(r^{2} f(r)+a^{2}\right)}\left[a(f(r)-1)\left(L_{1}+L_{2}\right) r^{2}-a^{2}(f(r)-3) r^{2}-L_{1} L_{2} f(r) r^{2}+(1+f(r)) r^{4}\right. \\
& -\sqrt{-r^{2}\left((f(r)-1) a^{2}-2 a(f(r)-1) L_{1}-r^{2}+f(r)\left(L_{1}^{2}+r^{2}\right)\right)} \\
& \sqrt{\left.-r^{2}\left((f(r)-1) a^{2}-2 a(f(r)-1) L_{2}-r^{2}+f(r)\left(L_{2}^{2}+r^{2}\right)\right)\right]} .
\end{aligned}
$$

\section{A. Near horizon collision in extremal rotating ABG black hole}

Note that for an extremal BH, the two horizons always coincides and located at $r=r_{e}$, where $r_{e}$ is the double root of Eq. (4), i.e., $\Delta\left(r_{e}\right)=r_{e}^{2} f\left(r_{e}\right)+a^{2}=0$.
We now study the properties of CM energy Eq. (20) as radius $r \rightarrow r_{e}$ for the extremal rotating ABG spacetime. Firstly we find the range of angular momentum of particles with which particles can reach the horizon by solving Eq. (13) numerically. The maximum/minimum angular momentum we are looking for extremal $\mathrm{BH}$ are listed in 
TABLE I: Numerical values of $\max / \min$ angular momentum for extremal rotating $\mathrm{ABG} \mathrm{BH} M=1, m_{0}=1, E=1$.

\begin{tabular}{llllll}
\hline \hline S.No. $Q$ & $a$ & $r_{e}$ & $L_{\min }$ & $L_{\max }$ \\
\hline 1 & 0.1 & 0.980186499171 & 1.01388 & -4.80898 & 2.02893 \\
2 & 0.2 & 0.922475716730 & 1.04536 & -4.75087 & 2.10710 \\
3 & 0.3 & 0.829553798960 & 1.07673 & -4.65290 & 2.22711 \\
4 & 0.4 & 0.700928370560 & 1.09464 & -4.50855 & 2.41043 \\
5 & 0.5 & 0.527525079404 & 1.08802 & -4.29774 & 2.71481 \\
\hline \hline
\end{tabular}

TABLE II: Numerical values of $\max / \mathrm{min}$ angular momentum for non-extremal rotating ABG BH $M=1, m_{0}=1, E=1$.

\begin{tabular}{llllll}
\hline \hline S.No. $Q$ & $a$ & $r_{+}$ & $L_{\min }$ & $L_{\max }$ \\
\hline 1 & 0.1 & 0.9 & 1.39848 & -4.75125 & 2.58660 \\
2 & 0.2 & 0.8 & 1.49272 & -4.66002 & 2.77108 \\
3 & 0.3 & 0.7 & 1.50591 & -4.63034 & 2.52549 \\
4 & 0.4 & 0.6 & 1.44629 & -4.42525 & 2.90270 \\
5 & 0.5 & 0.5 & 1.25836 & -4.27280 & 2.84983 \\
\hline \hline
\end{tabular}

Table (I). We can see from the Table (I) that when charge $Q$ is increases, the extremal horizon $r_{e}$ is also increases.

We can see the behavior of CM energy for extremal BH from Fig. 8 and Fig. 10, The Fig. 8 shows the variation of $E_{C M}$ vs $r$ for different values of $L_{1}$ and $L_{2}$ with fixed values of spin parameter $a$ and charge $Q$. We can see that $E_{C M}$ is infinite at the event horizon when $L_{1}$ is critical while for any other values of $L_{1}, E_{C M}$ is finite. In Fig. 10] we show the variation of $E_{C M}$ vs $a$ at the horizon for different values of $L_{1}$ and $L_{2}$ with fixed value of charge parameter $Q$.

\section{B. Near horizon collision in non-extremal rotating ABG black hole}

A BH is called non-extremal when the outer and inner horizons are not coincide, i.e. $r \neq r_{e}$. We find the range of angular momentum of particles by solving Eq. (13) numerically. The maximum/minimum angular momentum we are looking for non-extremal $\mathrm{BH}$ are listed in Table (III).

We can see the behavior of CM energy for non-extremal BH from Fig. 9 and Fig. 11 In Fig. 9 we show the variation of $E_{C M}$ vs $r$ for different values of $L_{1}$ and $L_{2}$ with fixed values of spin parameter $a$ and charge $Q$. Here, we can see that $E_{C M}$ is finite at the event horizon. In Fig. 11] we show the variation of $E_{C M}$ vs $a$ at the outer horizon for different values of $L_{1}$ and $L_{2}$ with fixed values of charge $Q$. The variation of $E_{C M}$ vs $r$ for different values of $Q$ can be seen in panel (a) of Fig. 12 and $E_{C M}$ vs $a$ in panel (a) of Fig. 13. We have also plot the variation of $E_{C M}$ for Kerr BH in panel (b) of Fig. 12 and in panel (b) of Fig. 13 .

Eq. (20) confirms that the non-linear charge $Q$ indeed has influence on the CM energy. In the limit $a \rightarrow 0$, Eq. (20) reduces to expression for $E_{C M}$ of $\mathrm{ABG} \mathrm{BH}$,

$$
\frac{E_{C M}^{2}}{2 m_{0}^{2}}(a \rightarrow 0)=\left[1+\frac{1}{f(r)}-\frac{L_{1} L_{2}}{r^{2}}-\frac{1}{f(r)} \sqrt{1-f(r)\left(1+\frac{L_{1}^{2}}{r^{2}}\right)} \sqrt{1-f(r)\left(1+\frac{L_{2}^{2}}{r^{2}}\right)}\right]
$$

which is exactly same as obtained in 23]. Further, $E_{C M}$ for the Kerr BH [1] can be obtained by taking limit $Q \rightarrow 0$ in Eq. (20), which reads

$$
\begin{aligned}
\frac{E_{C M}^{2}}{2 m_{0}^{2}}(Q \rightarrow 0) & =\frac{1}{r\left(r^{2}-2 r+a^{2}\right)}\left[2 a^{2}(1+r)-2 a\left(L_{1}+L_{2}\right)-L_{1} L_{2}(-2+r)+2(-1+r) r^{2}\right. \\
& \left.-\sqrt{2\left(a-L_{1}\right)^{2}-L_{1}^{2} r+2 r^{2}} \sqrt{2\left(a-L_{2}\right)^{2}-L_{2}^{2} r+2 r^{2}}\right]
\end{aligned}
$$

It turns out that Eq. (22) the $E_{C M}^{2}$ blows up at the horizon in the extremal case [1].

\section{CONCLUSION}

We are far from a reliable candidate for a quantum theory of gravity, hence recently more attention is given for phenomenological approaches to somehow solve these sin- 
gularity problem in classical general relativity and study possible implications. In this context, an important line of research is represented by the work on the regular $\mathrm{BH}$ solutions. Hence, we have investigated the collision of two particles falling freely from rest at infinity in the background of a rotating regular ABG BH. It was demonstrated by BSW [1] that CM energy of two colliding particles near the horizon of an extremal Kerr BH can be arbitrarily high value under some restriction on the angular momentum of particles. The analysis of BSW when extended to rotating $\mathrm{ABG} \mathrm{BH}$ to investigate the $\mathrm{CM}$ energy $E_{C M}$ for two particles, an unlimited $E_{C M}$ that constraints on the charge $Q$ and rotation parameter $a$. From the point of view of BSW mechanism the CM energy for two colliding particles for extremal $\mathrm{BH}$ should be arbitrarily high. So that we have found critical value of the angular momentum for which the CM energy of colliding particles is arbitrarily high near the horizon (extremal $\mathrm{BH})$. We have also seen that when the angular momentum of the colliding particles is not critical (non-extremal $\mathrm{BH}$ ) the CM energy is finite. For the non-extremal case, we find that the CM energy $E_{C M}$ increases with increase in the charge $Q$. The range of the angular momentum $L$ and the spin parameter $a$ increases for the rotating ABG $\mathrm{BH}$ as compared with the Kerr-Newman BH. The CM energy $E_{C M}$ for the extremal rotating $\mathrm{ABG} \mathrm{BH}$ case is infinite at $r_{e}=1.01388$ while for the extremal Kerr BH case the CM energy $E_{C M}$ is infinite at $r_{e}=1$.
Indeed, to obtain an arbitrary high $E_{C M}$, besides the conditions that the $\mathrm{BH}$ is an extremal $\mathrm{BH}$, and one of the particle has critical angular momentum, one still has restriction on the charge $Q$. It may be pointed out that horizon structure of the rotating $\mathrm{ABG} \mathrm{BHs}$ is significantly complicated to analyze analytically and hence we turn to numerically methods to analyze $E_{C M}$, the range of the angular momentum, with which particle can reach horizon and fall into $\mathrm{BH}$.

It turns out that if the angular momentum of a particle lies outside the range, it will not fall into the $\mathrm{BH}$ and no collision will take place. We have also estimated the $E_{C M}$ for non-extremal rotating $\mathrm{ABG}$ BH case, and found that the $E_{C M}$ is always finite and significantly affected by charge $Q$ and rotation parameter $a$. It will be of interest to extend the analysis discussed to other regular $\mathrm{BH}$, which will be determine whether these properties of regular BHs are generic. These and related work are subject of forthcoming papers.

\section{Acknowledgments}

M.A. acknowledges University Grant Commission, India for financial support through Maulana Azad National Fellowship scheme (File No.: F1-17.1/2012-13/MANF2012-13-MUS-RAJ-8679).
[1] M. Banados, J. Silk and S. M. West, Phys. Rev. Lett. 103, 111102 (2009).

[2] T. Jacobson and T. P. Sotiriou, Phys. Rev. Lett. 104, 021101 (2010).

[3] K. S. Thorne, Astrophys. J. 191, 507 (1974).

[4] K. Lake, Phys. Rev. Lett. 104, 211102 (2010).

[5] O. B. Zaslavskii, JETP Lett. 92, 571 (2010) [Pisma Zh. Eksp. Teor. Fiz. 92, 635 (2010)].

[6] S. W. Wei, Y. X. Liu, H. Guo and C. EFu, Phys. Rev. D 82, 103005 (2010).

[7] T. Igata, T. Harada and M. Kimura, Phys. Rev. D 85, 104028 (2012)

[8] C. Liu, S. Chen, C. Ding and J. Jing, Phys. Lett. B 701, 285 (2011).

[9] P. J. Mao, L. Y. Jia, J. R. Ren and R. Li, arXiv:1008.2660 [hep-th].

[10] I. Hussain, Mod. Phys. Lett. A 27, 1250017 (2012).

[11] J. Yang, Y. L. Li, Y. Li, S. W. Wei and Y. X. Liu, arXiv:1202.4159 [hep-th].

[12] Y. Zhu, S. -F. Wu, Y. -X. Liu and Y. Jiang, Phys. Rev. D 84, 043006 (2011).

[13] A. A. Grib and Y. .V. Pavlov, Grav. Cosmol. 17, 42 (2011).

[14] A. A. Grib, Y. .V. Pavlov, O. F. Piattella and O. F. Piattella, Int. J. Mod. Phys. A 26, 3856 (2011).

[15] A. A. Grib and Y. .V. Pavlov, Astropart. Phys. 34, 581 (2011).

[16] A. A. Grib and Y. .V. Pavlov, Europhys. Lett. 101, 20004 (2013).
[17] O. B. Zaslavskii, Phys. Rev. D 82, 083004 (2010).

[18] O. B. Zaslavskii, Phys. Lett. B 712, 161 (2012).

[19] O. B. Zaslavskii, Class. Quant. Grav. 28, 105010 (2011).

[20] O. B. Zaslavskii, Int. J. Mod. Phys. D 22, 1350044 (2013).

[21] M. Patil and P. S. Joshi, Class. Quant. Grav. 28, 235012 (2011).

[22] M. Patil, P. S. Joshi, M. Kimura and K. i. Nakao, Phys. Rev. D 86, 084023 (2012).

[23] P. Pradhan, arXiv:1402.2748 [gr-qc].

[24] B. Toshmatov, B. Ahmedov, A. Abdujabbarov and Z. Stuchlik, arXiv:1404.6443 [gr-qc].

[25] J. Bardeen, in Proceedings of GR5, Tiflis, U.S.S.R., (1968).

[26] E. Ayon-Beato and A. Garcia, Phys. Rev. Lett. 80, 5056 (1998).

[27] S. A. Hayward, Phys. Rev. Lett. 96, 031103 (2006).

[28] C. Bambi and L. Modesto, Phys. Lett. B 721, 329 (2013).

[29] R.P. Kerr, Phys. Rev. Letters, 11,237 (1963).

[30] K. Schwarzschild, Uber das Gravitationsfeld eines Mapunktes nach der Einsteinschen Theorie, Sitzber. Deutsch. Akad. Wiss. Berlin, Kl. Math. Phys. Tech., 189, (1916).

[31] E. Ayón-Beato, A. García, Phys. Lett. B 464, 25 (1999).

[32] C. Bambi, Mod. Phys. Lett. A 26, 2453 (2011).

[33] C. Bambi, Astron. Rev. 8, 4 (2013).

[34] S. N. Zhang, W. Cui and W. Chen, Astrophys. J. 482, L155 (1997).

[35] J. M. Bardeen, W. H. Press and S. A. Teukolsky, Astro- 
phys. J. 178, 347 (1972). 